\title{
La atención Temprana, Ayer, Hoy y Mañana. Derechos, un Lugar desde el que Partir, un Lugar hacia el que Mirar
}

\section{Early Childhood Intervention, Yesterday, Today and Tomorrow. Rights, a Place of Departure and a Place to aim Toward}

\author{
José Antonio Luengo Latorre \\ Universidad Camilo José Cela, España
}

\begin{abstract}
Resumen. La atención temprana no es solo un concepto. Ni siquiera una práctica. La atención temprana representa un proceso ligado al desarrollo de patrones y protocolos para la promoción de la salud, en el más amplio sentido de la palabra, en los primeros años de vida. Y, consecuentemente, supone, debería suponer, el desarrollo de un derecho, habilitado en intervenciones planificadas, técnicamente consecuentes y adecuadamente combinadas, entre profesionales e instituciones, para el tratamiento y el abordaje de las situaciones que son inherentes a circunstancias de discapacidad o riesgo de su aparición en la población de más corta edad. El objetivo es abundar en la acción proactiva, la tarea preventiva, la escucha y la comunicación con el entorno familiar, la agilización de los trámites, la evitación de los efectos perversos de la reiteración y repetición de pruebas, consultas, comprobaciones, la comunicación certera y eficaz entre los profesionales y sistemas implicados, la ausencia de arrogancia y protagonismo institucional.

Palabras clave: Atención temprana, Derechos.
\end{abstract}

\begin{abstract}
Early attention is not just a concept. It is not even simply a practice. Early attention is a process linked to the development of standards and protocols for promoting health in the broadest sense of the word, in the first days of life. Consequently it is, or should mean, the development of a right, enabled through planned interventions which are technically consistent and properly combined, between professionals and institutions, to deal with situations inherent to circumstances of disability or the risk of the appearance of disability, at an early age. The objective is to develop proactive measures, preventative work, listening and communication in the family. Of equal importance are the streamlining of procedures, avoiding the negative effects of the repetition of tests, consultations, checks, accurate and effective communication between professionals and systems and institutions that do not arrogantly try to take the leading role.
\end{abstract}

Keywords: Early attention, rights.

\section{A modo de introducción: lo que se sabe, lo que se hace}

Si algo hemos aprendido sobre la atención temprana desde hace más de treinta años es que se trata, básicamente, de un marco de intervención sistémico, multidisciplinar y иbicuo, que se desarrolla al mismo tiempo en diferentes espacios y, de modo singular, con la participación directa de profesionales de muy diferentes ámbitos disciplinares y dependencias institucionales y con el protagonismo esencial del entorno familiar y el contexto natural que rodea al sujeto de derechos que es el niño (Peterander, 2009). A diferencia de lo que puede llegar a pensarse, se trata, pues, de

José Antonio Luengo Latorre, además de ser profesor en la Universidad Camilo José Cela, es Especialista en Atención Temprana. La correspondencia sobre este artículo debe enviarse al autor al e-mail: joseantonioluengo@gmail.com una manera de concebirla que huye del monopolio, de la exclusividad de uso por parte de los profesionales o de determinadas instituciones frente a otras. Muy al contrario, los años transcurridos han definido más bien un recorrido en el que la sindicación de fuerzas, la ausencia de protagonismos estériles, la comunicación entre profesionales e instituciones y, sobre todo, la relevancia del entorno familiar en la configuración y desarrollo de los programas a implementar son (o deberían ser), de manera sustantiva, ejes nucleares de la intervención. En fin, nada que no se lleve diciendo desde hace mucho, mucho tiempo. Nada que algunos no hayamos experimentado en nuestra particular experiencia profesional. El sistema al servicio del usuario. Los tiempos, los procesos, las herramientas y estrategias al servicio de las necesidades y de los derechos de los afectados, nunca al revés. La acción proactiva, la tarea preventiva, la escucha y la comunicación con el entorno familiar, la agilización de los trá- 
mites, la evitación de los efectos perversos de la reiteración y repetición de pruebas, consultas, comprobaciones, la comunicación certera y eficaz entre los profesionales y sistemas implicados, la ausencia de arrogancia y protagonismo institucional. El valor de los usuarios, de los niños y sus familias. El valor de su presente, de su futuro, claro. Pero, principalmente, de su presente. Ese el primero. Su primer derecho. El derecho a vivir de una manera digna, con calidad de vida (Giné, 2009), desde su nacimiento, desde sus primeras miradas, sus primeros contactos con un mundo nuevo, en muchas ocasiones hostil. Su derecho a estar y ser de la manera más saludable, viva e intensa. El derecho de sus padres y familia a saber, a conocer, a opinar, a decidir y sentir, a tropezar y levantarse, a equivocarse y a buscar respuestas. El derecho a actuar desde el primer momento. El derecho a que se actúe desde el primer momento. Y con la participación e implicación protagónicas del entorno. La intervención temprana entendida como "un servicio para niños muy pequeños con necesidades especiales y sus familias para asegurar y garantizar el desarrollo personal del niño, fortalecer las competencias de las familias y promover la integración social del niño y de su familial". No sé si estamos suficientemente cerca de este modelo.

Los padres y el entorno próximo definen su experiencia. Existe, dicen, un mundo desconocido, cargado de terminología indescifrable, miradas inseguras, perspectivas impredecibles, incertidumbre. Un mundo plagado de tecnicismos, vacío y frío. Un mundo de sufrimiento, sí, pero también de lucha, de esfuerzo, de afecto y amor. Un mundo de entrega y abnegación. Un mundo marcado por, probablemente, una de las experiencias más traumáticas a las que puede enfrentarse un ser humano. Algo no ha salido bien. Tenemos que hacer más pruebas pero su hijo tiene algún problema... Un mundo de peregrinajes y búsqueda. Al final, casi siempre, la vida, en sentido amplio, se abre camino. Busca su espacio, su recorrido, utiliza sus tiempos, sus propios registros y parámetros de desarrollo. Y se expande. Se equilibra, se afianza. Busca su sitio. Y normalmente lo encuentra. Al final el esfuerzo de los que viven la experiencia establece un ritmo, una secuencia, un camino para la adaptación, para la creación de un nuevo panorama en el que crecer, depositar las esperanzas y crecer, llorar y crecer, aprender y crecer. El equilibrio vuelve, la lucha y el trabajo, el esfuerzo denodado persiste, pero la idea de que es posible vivir vuelve, renace, se recompone, se alza entre ruinas. $\mathrm{Y}$ todo empieza un día. Un diagnóstico, un pronóstico, una idea de lo que la vida se ha llevado y ha traído. Un recorrido, un contexto poliédrico.

\footnotetext{
${ }^{1}$ Peterander, (2009). Texto citado.
}

\section{El contexto de la atención temprana, el camino recorrido, algunos hitos}

Este es el contexto en el que se ubica, de forma explícita, la atención temprana. No solo, pero éste es esencial. Una experiencia traumática de los que son o serán nuevos usuarios de un sistema que, ligado a la experiencia singular de tener un hijo en condiciones nunca esperadas, pretende afrontar el siempre complejo reto de trazar un camino mejor, predecible, susceptible a mejoras permanentes, innovador y, por qué no decirlo, amable. Un entorno, profesional y humano, que surge de las dificultades, duras e incisivas, derivadas de la duda que no cesa, de la incertidumbre lacerante, o, también, a veces de forma definitiva, de las costosas certidumbres ${ }^{2}$.

El diagnóstico definitivo, la idea de futuro tumbada, lastrada, aletargada por una idea inasumible, por la inseguridad, por la liquidación de todo lo imaginado, muy prudentemente, durante los meses de gestación. Se escapa la idea del hijo que iba a nacer, crecer y vivir. Se esfuma la idea que amanecía cada mañana al pensar en quien iba a nacer en poco tiempo, quien iba a ocupar un espacio nuevo en la casa, en la mente y en la vida de quienes esperaban el feliz acontecimiento de ver nacer. Meses esperando la mejor noticia, y bastan unos segundos para que la experiencia estalle en mil pedazos. Segundos tan solo para hacer frente a una idea nueva, a una imagen diferente, a un recorrido no pensado, ni siquiera imaginado. Este es el sitio, el espacio en el que ha de crecer la adecuada respuesta técnica profesional e institucional. Un mundo, toda una vida por delante, para miles de niños. Nacen en shock, en precario, especialmente vulnerables, inmersos en un proceso que ha de construirse, siempre, en el marco y contexto de sus necesidades, las de ahora, las que se ven y perciben y las que no, las que se observan y las que se intuyen, las que están y las que podrán llegar. Pero también, y esto es esencial, un proceso que ha de desplegarse en el tejido de sus derechos, por encima de todo lo demás. Porque el futuro para todos estos niños y sus familias no es mañana. Es hoy, precisamente hoy. Hoy es el día. Hoy es cuando hay que actuar, hoy cuando hay que hacer, trabajar, apoyar, enseñar. Hoy es el día. Actuar rápida, profesional y diligentemente. Mañana no existe. Porque actuar hoy cambiará su mañana. Y el de muchos que le rodean. Y el nuestro como sociedad. Porque si así lo hacemos, habremos dado pasos para cambiar sustancialmente la vida de muchas, muchas personas. Y de eso estamos hablando.

\footnotetext{
2 http://www.facebook.com/pages/Una-posibilidad-entre-mil/97607 598487. "Una posibilidad entre mil" es una historia autobiográfica. Cristina y Miguel Ángel son padres por primera vez, pero el futuro de la pequeña Laia está en peligro desde los primeros instantes. Hospitales, centros de rehabilitación y médicos se convierten entonces en su rutina diaria. Hay una posibilidad entre mil de que Laia salga adelante. Y se agarra a ella con una fuerza tremenda, una alegría desbordante, arrastrando a todos por el camino de la vida.
} 
Ser o no ser, una frase que, aquí, adquiere también valor.

La atención temprana ha cambiado mucho, se dice, en todos estos años. ¿O no tanto? De esto habría que hablar mucho y no es fácil sintetizar una respuesta. Hay cosas, entiendo, que no han modificado excesivamente sus recorridos. Los conceptos, eso sí, han ido acuñándose, depurándose, confirmándose. Parece existir consenso suficiente. Hemos acordado un concepto esencial. "Se entiende por Atención Temprana el "conjunto de intervenciones, dirigidas a la población infantil de 0 a 6 años, a la familia y al entorno, que tienen por objeto dar respuesta lo más pronto posible a las necesidades transitorias o permanentes que presentan los niños con trastornos en su desarrollo o que tienen el riesgo de padecerlos. Estas intervenciones, que deben considerar la globalidad del niño, han de ser planificadas por un equipo de profesionales de orientación interdisciplinar o transdisciplinar" 3 .

Experiencias de trabajo conjunto ${ }^{4}$, debates profesionales, libros blancos y publicaciones específicas han ido acotando, asimismo, las ideas, los argumentos, la filosofía subyacente al propio proceso de definición e intervención. Contenidos de naturaleza nuclear han sido invocados en torno a ámbitos como (1) el protagonismo y la participación de y con las familias -intervención temprana y apoyo familiar mediante redes sociales formales e informales- (Dunst, 2005) y el rol de los técnicos y especialistas (Luengo, Torrego y García, 1994); (2) la integración social y familiar; (3) la gratuidad, universalidad e igualdad de oportunidades; (4) la interdisciplinariedad y alta cualificación profesional; (5) la descentralización y la sectorización de los recursos y servicios y (6) la coordinación y trabajo conjunto de los expertos y las instituciones de las que dependen (Luengo, 1995).

Con el paso de los años y la experiencia acumulada, se han descartado modelos y han surgido nuevos marcos para la intervención. Se han superado obstáculos y han surgido, también, nuevos retos. ¿Pero nos hemos movido en la dirección adecuada? Algunos hitos son fácilmente reconocibles en el tiempo. Hace ya tiempo del alumbramiento e implantación de algunos de ellos: (1) El desarrollo de planes y programas de prevención de minusvalías; (2) los programas de control y seguimiento neonatológico, especialmente en el ámbito de la intervención con prematuros; (3) el asentamiento de la etapa de educación infantil como etapa plenamente educativa y la extensión de las escuelas infantiles como contexto normalizador y marco esencial para la

\footnotetext{
${ }^{3}$ Federación estatal de asociaciones de profesionales de Atención Temprana. Libro Blanco de Atención Temprana. Documentos. 55/2005. Real Patronato sobre Discapacidad, 2005. Madrid.

${ }^{4}$ Debe destacarse especialmente, entre otros, el trabajo del Grupo PADI y Genysi en la Comunidad de Madrid, con especial significación al liderazgo profesional y moral ejercido por el Dr. José Arizcun Pineda, y de la Federación Estatal de Asociaciones de Profesionales de Atención Temprana.
}

compensación de desigualdades; (4) la implantación de servicios y recursos de atención temprana en el ámbito educativo; (5) el desarrollo del modelo de integración de alumnos con necesidades educativas especiales en la escuela ordinaria. Y, por supuesto, (6) la extensión de los programas y servicios de atención temprana.

$\mathrm{Y}$ es evidente, se han dado pasos y no poco sustantivos. Sin embargo, no podemos, ni debemos, mirar hacia ese lado exclusivamente. Atrás quedan ideas y proyectos que no han terminado de cuajar y, abundando en la misma idea, encontramos espacios de trabajo e intervención que apenas han evolucionado, dejando al aire observaciones críticas y de mejora que cuentan con más de veinte años de presencia sin que se haya ahondado suficientemente en su abordaje. Al menos con éxito. La experiencia actual nos sitúa en un modelo excesivamente fragmentado, huidizo de la visión integral que sería deseable. Un modelo alimentado por la suma de actuaciones, no por la integración de las mismas. Lastrado por la acción compartimentada, y, a modo de entender de quien suscribe, excesivamente orientada por la óptica profesional, alejada del, entiendo, imprescindible orden, criterio y norte que ha de surgir del propio entorno familiar. Un entorno, deberíamos pensar, con capacidad para entender, dirigir, hacer, reorientar; y un contexto profesional centrado en las necesidades y derechos del sistema con el que se trabaja, con el que se comparte, con el que se aprende, con el que se crece y al que se ayuda a crecer.

Existen, al menos, tres claves que siempre habrán de ser abordadas con cordura, sensatez y convencimiento para hacer frente de manera razonable al desarrollo sostenible de un modelo de atención temprana satisfactorio. Responderían a tres preguntas básicas: por qué intervenir, cuándo y cómo. (1) El reconocimiento de los derechos de los usuarios (el tránsito de las necesidades a los derechos); (2) la intervención profesional y rápida para todos los que lo necesitan (actuar bien hoy, y no mañana, con todos) y, por supuesto, la actuación combinada de instituciones y agentes (la verdadera acción interinstitucional, ceder "poder" para que gane consistencia el protagonismo del sistema usuario).

Es en estos parámetros en los que ha de asentarse la idea y la práctica de la atención temprana que queremos. Y este escenario de objetivos y ámbitos para la mejora no es, desgraciadamente, tan diferente al que dibujábamos hace algunos años. Porque, insisto, hay cosas en las que se ha evolucionado poco. Y algunas, por desgracia, no han ido a mejor. A estas claves dedicaré las reflexiones esenciales del presente texto.

\section{El niño, sujeto de derechos}

Probablemente la infancia nunca ha estado tan defendida y amparada jurídicamente como en el 
momento actual. De modo singular, desde la Convención de los derechos de la infancia en 1989, ratificada por el Estado español en 1990, el tejido y cuerpo normativo ha venido incorporando sus preceptos y consideraciones en el ordenamiento jurídico que regula la vida de los ciudadanos y, en especial, la de la infancia y la adolescencia. Los niños, hoy, en el ámbito jurídico al menos, no son definidos desde sus carencias, por lo que les falta para ser adultos; se les define por sus atributos, por su presente, por sus posibilidades. Se considera a cada niño como un sujeto pleno de derechos exigibles. Hace tiempo que dijimos adiós, pues, a la visión del niño como un conjunto de necesidades, objeto de protección y control de los padres o el Estado, contexto social y normativo que fue el eje de la llamada legislación de menores en el mundo entero. Desde el punto de vista jurídico entendemos por derecho la facultad del ser humano para hacer legítimamente lo que conduce a los fines de su vida... y para hacer o exigir todo aquello que la ley o la autoridad establece en su favor. Desde una perspectiva más vivencial, podríamos concebirlo como una experiencia vital personal y estable que nos permite afrontar la vida (en todas o en alguna de sus manifestaciones) con garantías, cuidado y atención externos, medios y recursos. No hablamos, pues, de cualquier cosa. Es una facultad que tenemos; es casi un tesoro que nos permite estar y ser. Pero, para ello, debe existir una norma que especifique claramente el cuerpo de opciones que atesoramos, las garantías con las que contamos para hacer, ser, disponer, o tener.

Del derecho objetivo, o norma, nace el derecho subjetivo como facultad, que se expresa cuando se dice, que derecho es aquello que me es lícito o permitido hacer; consecuentemente, hablamos de "un atributo, una facultad o una situación de poder que una persona posee, otorgado por una norma jurídica que le protege y tutela, ya que, en caso de ser vulnerado, los órganos jurisdiccionales pueden exigir su efectividad" (Cruz, 1998). El derecho subjetivo nos protege, nos tutela, nos da garantías.

La Constitución Española (CE) concreta y clasifica los deberes y derechos fundamentales de todos los ciudadanos en su Título Primero, y, de modo singular, en los Capítulos II y III de aquel. Y lo hace planteando una suerte de jerarquía que define diferencias no poco importantes para su exigencia en caso de duda sobre su hipotética vulneración. Capítulo II. Sección Primera: Derechos fundamentales y libertades públicas (artículos 15 al 29); Sección segunda: De los derechos y deberes de los ciudadanos (artículos 30 al 38) y Capitulo III: De los Principios rectores de la Política Social y Económica (artículos 39 al 52). Los derechos y libertades citados en el primer grupo, a saber, derechos civiles y políticos de fundamental relevancia tales como, entre otros, el derecho a la vida y a la integridad física y social, a la libertad ideológica y religiosa, al honor, a la intimidad personal y familiar y a la propia imagen, incluyen también algunos derechos sociales relevantes como el derecho a la educación, por ejemplo, vinculan a todos los poderes públicos, son inmediata y directamente aplicables y están tutelados mediante la posibilidad de un recurso de amparo ante el Tribunal Constitucional (artículo 53 de la CE). Por su parte, los derechos citados en el segundo grupo (entre los que se encuentran, por ejemplo, el derecho a contraer matrimonio con plena igualdad jurídica, el derecho a la propiedad privada o el derecho al trabajo y a una vivienda digna) también vinculan a los poderes públicos y son exigibles, si bien no gozan del procedimiento especial ni del recurso de amparo antes citado. Por último, los derechos reseñados en el tercer grupo, que recoge de manera manifiesta la mayor parte de los derechos de naturaleza social, informan sobre la legislación positiva, la práctica judicial y la actuación de los poderes públicos, pero no los vincula ni son directa e inmediatamente exigibles y solo pueden ser alegados ante la jurisdicción ordinaria de acuerdo con lo que dispongan las leyes que lo desarrollen. En concreto, los artículos $43^{5}$ y $49^{6} \mathrm{de}$ la CE, relativos a la protección de la salud y a la atención a las personas con discapacidad, están incluidos en este grupo. Por tanto, nos encontramos con derechos de diferentes velocidades, recorrido y aliento. En el citado grupo tercero, el que afecta de manera específica a contenidos como familia e infancia, discapacidad, promoción de la salud y prevención de las enfermedades, encontramos el bloque de derechos-principios rectores de mayor fragilidad, cuya aplicación queda supeditada de manera expresa a los que dicten las leyes sobre su desarrollo (Vilá, 2009).

Por tanto, de lo reseñado y en lo que atañe a los contenidos que nos son de referencia, cabe plantearse que nos encontramos con derechos constitucionales cuya concreción se haya supeditada de manera notable al desarrollo de políticas específicas, siempre en función de parámetros presupuestarios $\mathrm{y}$, consecuentemente, con las limitaciones derivadas de las circunstancias y modos de interpretación de las diferentes realidades que pueden asociarse a lineamientos ideológicos o coyunturas macroeconómicas. La idea, pues, está clara. La CE marca los escenarios y las leyes correspondientes, $\mathrm{y}$, por supuesto, sus normas de desarrollo (Reales Decretos, Decretos, Órdenes, Instrucciones, etc.) definen con posterioridad el grado de concreción de los derechos y deberes referenciados. Iniciamos, a partir de este marco de recorrido jurídico un espacio en

\footnotetext{
${ }^{5}$ Se reconoce el derecho a la protección de la salud. Compete a los poderes públicos organizar y tutelar la salud pública a través de medidas preventivas y de las prestaciones y servicios necesarios. La ley establecerá los derechos y deberes de todos al respecto.

${ }^{6}$ Los poderes públicos realizarán una política de previsión, tratamiento, rehabilitación e integración de los disminuidos físicos, sensoriales y psíquicos, a los que prestarán la atención especializada que requieran y los ampararán especialmente para el disfrute de los derechos que este Título otorga a todos los ciudadanos.
} 
que, tal como se ha especificado, determinadas situaciones coyunturales, de diferente naturaleza, pueden suponer un marco de desarrollo programático y de ejercicio de derechos efectivos más o menos ambicioso en temas cuya regulación jurídica se define por su excesiva amplitud, ambigüedad o indefinición.

La Atención Temprana representa de modo específico un buen ejemplo de lo expuesto. Sin ser exhaustivos, por razón de objetivos del presente texto y su extensión, la legislación estatal que ha venido regulando los derechos reseñados en el grupo tercero antes citado y, en concreto, la que afecta a la Atención Temprana y sus relaciones con los ámbitos de promoción de la salud, prevención y atención de y $a$ la discapacidad (en sus ámbitos educativos y de abordaje terapéutico y de tratamiento específico) -podríamos hablar, en todo caso, de prevención primaria, secundaria y terciaria en los diferentes contextos de atenciónno ha definido de manera explícita la Atención Temprana como contenido esencial de derecho, más allá de las referencias a ámbitos como la ordenación de las actuaciones en materia de prevención, al consejo genético y diagnóstico precoz y a la asistencia pediátri$\mathrm{ca}$, o a las prestaciones sanitaria en general, la rehabilitación y los servicios sociales, reflejados en el Título III de la Ley de Integración Social de los Minusválidos (LISMI, 1982); o a núcleos temáticos como la atención al alumnado con necesidades educativas especiales y su escolarización, detallados en los artículos 73 y 74 de la Ley Orgánica 2/2006 de Educación (LOE); o a los ámbitos de actuación fijados por el Capítulo II, sobre las actuaciones sanitarias del sistema de salud de la Ley General de Sanidad, de 1986) o, entre otros apartados, a lo establecido en el artículo 14 de la Ley de Cohesión y Calidad del Sistema nacional de Salud (2003): se define al prestación socio-sanitaria desde el sistema sanitario como "el conjunto de cuidados destinados a aquellos enfermos, generalmente crónicos, que por sus especiales características pueden beneficiarse de la actuación simultánea y sinérgica de los servicios sanitarios y sociales para aumentar su autonomía, paliar sus limitaciones o sufrimientos y facilitar su reinserción social".

Mención especial requiere la Ley 39/2006, de 14 de diciembre, de Promoción de la Autonomía Personal y Atención a las personas en situación de dependencia, que tiene por objeto regular las condiciones básicas que garanticen la igualdad en el ejercicio del derecho subjetivo de ciudadanía a la promoción de la autonomía personal y atención a las personas en situación de dependencia, en los términos establecidos en las leyes, mediante la creación de un Sistema para la Autonomía y Atención a la Dependencia (SAAD), con la colaboración y participación de todas las Administraciones Públicas y la garantía por la Administración General del Estado de un contenido mínimo común de derechos para todos los ciudadanos en cualquier parte del territorio del Estado español. La ley regula el derecho de acceso a la prestaciones (hablaríamos de un derecho subjetivo) y faculta a los interesados y solicitantes a iniciar las acciones administrativas y judiciales que estimen en función del derecho que le reconoce la Ley. También regula ésta los derechos y deberes de las personas en situación de dependencia.

Tras un amplio debate sobre la pertinencia de tomar en consideración las necesidades derivadas de la atención a la población entre cero y tres años ${ }^{7}$, la citada Ley introdujo en su disposición adicional decimotercera la Protección de los menores de tres años, ratificando de este modo la concreción de las necesidades de la población afectada de necesidades de ayuda a domicilio y, en su caso, de prestaciones económicas vinculadas y para cuidados en el entorno familiar a favor de los menores de tres años acreditados en situación de dependencia. En cualquier caso, hacemos referencia a un marco normativo que ampara las necesidades del entorno relacionadas de manera objetiva con las implicaciones sustantivas en el cuidado especial a los menores de tres años afectados de algún tipo de discapacidad. Y si bien hablamos de una norma que no aborda de manera directa el derecho a una atención específica y temprana por razón de discapacidad en los tres primeros años de vida, sí invoca de manera singular la contribución en forma de diferentes tipos de ayudas a las familias a la ya citada necesidad de dar respuesta lo más pronto posible a las necesidades transitorias o permanentes que presentan los niños con trastornos en su desarrollo o que tienen el riesgo de padecerlos. Esta norma define, pues, al acceso a los servicios y prestaciones del Sistema para la Autonomía y Atención a la Dependencia, en forma de ayudas económicas vinculadas, como ya se ha especificado, y para los cuidados en el entorno familiar, todo ello, sin perjuicio de los servicios educativos y sanitarios que correspondan. Y prevé, asimismo, sin obviar sus competencias para facilitar los servicios de atención temprana y rehabilitación, la promoción por parte del Consejo Territorial del SAAD de un plan de atención al colectivo de menores de tres años en el que se contemplen las medidas a adoptar por las administraciones públicas. Amén de las dificultades observadas para la definición y concreción del referido plan, las circunstancias negativas que rodean la aplicación de esta ley se han visto específicamente relacionadas con el tiempo transcurrido entre la solicitud y la resolución por parte de las administraciones y, por supuesto, con el grado de acuerdo en relación a la especificación de las citadas resoluciones a las expectativas e interpretación de necesidades reales expuestas por los solicitantes, en clara relación con la insatisfacción apreciada por éstos sobre los resultados de las valoraciones de la dependencia elaboradas por los servicios técnicos específicos y, consiguientemente, sobre las prestaciones definitivamente aprobadas.

\footnotetext{
${ }^{7}$ A partir de los tres años debe considerarse que los menores afectados pueden ser titulares de las prestaciones ajustadas a sus necesidades.
} 
No se trata, en cualquier caso, y es importante considerarlo, de un texto que haya regulado y, por tanto, zanjado, la Atención Temprana como concepto y marco de intervenciones. Ni es su objetivo, todo hay que decirlo. Pero no hay que obviar, que, además, regula un procedimiento de acceso a sus medidas protectoras que no parece adecuado para la protección activa. En palabras de Casado (2011) "la LAAD es norma de objetos parciales, sobre los que incide de modo en parte experto y en parte inexperto, y con un acceso problemático a la protección activa”. Sin obviar mejoras sobre los parámetros establecidos hasta su promulgación ${ }^{8}$, su desarrollo no ha contribuido precisamente a dar estabilidad y cobertura a las diferentes necesidades inherentes en el colectivo de afectados en el ámbito de la atención temprana, en el que la determinación de la propia necesidad se vincula de manera específica a situaciones no expresamente reconocidas por el sistema, como el caso de las coyunturas de riesgo sin concreción de discapacidad expresa. Y este escenario es especialmente relevante en el contexto que está siendo abordado. Hablaríamos así de la constatación de una línea de separación escasamente aceptable entre los niños que pudieran beneficiarse de los principios del derecho subjetivo (los afectados de algún tipo de discapacidad) y los que no (las situaciones de riesgo sin evidencia explícita de aquella) o en función del criterio de gravedad y no de necesidad.

Como bien conocemos, el ordenamiento jurídico define siempre su trayectoria normativa desde el corpus estatal a la secuencial actuación legislativa desarrollada por las diferentes Comunidades Autónomas en el ámbito de sus competencias y responsabilidades, contraídas en el marco de los respectivos estatutos de autonomía; y es en este contexto donde podemos encontrar una mayor regulación y concreción, según Comunidades, en el ámbito que nos ocupa. Las Comunidades Autónomas han ido regulando en materia de atención temprana en el espacio jurídico de la legislación de Servicios Sociales ${ }^{9}$.

En general, el análisis de la normativa autonómica en esta materia se ubica en el entorno de los principios establecidos por la ya citada Ley para la Promoción de la Autonomía Personal y Atención a las personas en situación de dependencia, a saber, universalidad, responsabilidad pública, igualdad, equidad, prevención, normalización autonomía, participación, atención per-

\footnotetext{
${ }^{8}$ La LAAD ha establecido la institución de derechos subjetivos para el acceso a la protección social de la que sólo gozaban en ese régimen de garantía los beneficiarios de prestaciones de la Seguridad Social por gran invalidez y similares (Casado, 2011)

${ }^{9}$ No es irrelevante el debate sobre si el contexto de protección social debe prevalecer sobre el sanitario y/o el educativo en la concreción de las políticas de desarrollo de las actuaciones esenciales en materia de atención temprana. Teniendo en cuenta las condiciones ya citadas de definición y ubicación jurídica en las que el ámbito de protección social se encuentra, claramente diversificada en los desarrollos legislativos autonómicos, no es de extrañar la falta de criterio uniforme de la que se adolece y a la que se hace referencia.
}

sonal e integral, coordinación y cooperación y calidad de servicios. Y del citado análisis no puede extraerse una conclusión especialmente favorable para la atención temprana. Y es difícil que encontremos otra lectura toda vez que adolecemos de un marco general de carácter estatal que contribuiría a dar equidad, cohesión, coherencia, sentido y verdadera igualdad a la respuesta de las administraciones, de todas las administraciones, a una necesidad, $y$, tal como se ha venido reseñando, a lo que entendemos como un derecho subjetivo que debería ser considerado irrefutable. Un derecho ligado a la prevención en materia sanitaria y educativa en sus diferentes ámbitos y recorridos, un derecho ligado a la dignidad y autonomía personal en sentido estricto, a la posibilidad de crecer y madurar con las máximas posibilidades, a la posibilidad de reducir de manera notable, cuando no eliminar, los riesgos derivados de determinadas situaciones definidas en el contexto de las alteraciones del desarrollo en los primeros momentos de la vida. En cualquier caso, la normativa estatal aporta suficientes elementos conceptuales como para que la respuesta, si tiene que ser diversificada, invoque, al menos, la condición de prestación garantizada a la atención temprana. Y ya encontramos líneas de abordaje satisfactorias que definen, en todo caso, un espacio para la mejora y el razonable optimismo.

La respuesta es diferente según Comunidades, en algunos casos de manera notable. Los conceptos jurídicos utilizados definen derechos garantizados de prestación del servicio en algunos casos, o referidos a prestaciones esenciales como derecho exigible en otros. Hablamos en estos supuestos de opciones legislativas que apuestan por la consideración de la atención temprana como un derecho subjetivo, superando de manera significativa la visión de los procesos desde la óptica de marcos normativos ligados esencialmente a la sujeción, a las posibilidades presupuestarias y a los principios de prelación y concurrencia.

Este es el camino. El reto, la apuesta por la consideración de la atención temprana como un derecho subjetivo, ligado a la prestación garantizada de un servicio de atención especializado e incorporado a un catálogo de servicios amplio, integral y coordinado. Y esta última condición se antoja imprescindible. Porque es imprescindible dotar de coherencia y sentido combinado a las actuaciones desarrolladas en los entornos sociales, educativos y sanitarios.

Amén de otras ideas y argumentos, lo expuesto permite realizar tres consideraciones de carácter relevante, a modo de síntesis, relacionadas con el presente apartado: (1) Los avances en materia de reconocimiento de derechos en el ámbito de la Atención Temprana no son escasos. En los últimos años, el ordenamiento jurídico ha evidenciado cambios relevantes. $\mathrm{Al}$ menos en la formulación de derechos y garantías. No obstante, estamos sólo rascando la superficie; nos hallamos en el origen de un cambio sustantivo que se entiende imprescindible. La atención temprana, como presta- 
ción de servicios irrenunciable, requiere de un sustrato jurídico estable y uniforme que habilite las cuotas necesarias de garantía y sostenibilidad de recursos y procesos tasados de intervención especializada e integral, en sus componentes de interdisciplinar e interinstitucional. Pensar en una concreción jurídica de ámbito estatal en el momento actual puede representar una entelequia. Sin embargo, sería deseable, y es necesario insistir: la conceptualización legislativa estatal habilita y permite la definición y regulación sólida en el marco del desarrollo normativo del que son responsables las Comunidades Autónomas. (2) En términos generales, la regulación de derechos y prestaciones de servicios en los ámbitos sanitario y educativo habilita un recorrido razonable, siempre susceptible de mejora, en el ámbito de desarrollo de las actuaciones prácticas con los niños susceptibles de atención y sus familias. Es el contexto social, en el que se encuentra ubicada de forma ordinaria la prestación de servicios y centros específicos de atención temprana, el escenario más necesitado de niveles sensibles de optimización, en los términos antes citados de dotación de recursos suficientes, sostenibles y uniformes. (3) Las actuaciones en contextos definidos como de riesgo, sin que medie especificación o diagnóstico de discapacidad, precisan de un soporte y estabilidad técnica y jurídica imprescindibles, siempre en el marco de las intervenciones derivadas de los protocolos definidos, disciplinar e institucionalmente en diferentes ámbitos doctrinales, para la prevención primaria y secundaria.

\section{La intervención rápida para todos los que lo nece- sitan y la acción coordinada}

Hoy es cuando hay que actuar, hoy cuando hay que hacer, trabajar, apoyar, enseñar y facilitar. Hoy es el día. Actuar rápida, profesional y diligentemente. El mañana, hoy, no existe. Pero actuar hoy definirá un mañana mejor. $\mathrm{Y}$ el de muchos que le rodean. $\mathrm{Y}$ el nuestro como sociedad. Y de eso estamos hablando. Hablamos de la necesidad de abordar la atención temprana como un derecho subjetivo, sí. Para todos los que la necesitan. Se juegan mucho. Nos jugamos mucho. Y con la mayor celeridad. El reto está ahí, en frente nuestro. Nos mira a los ojos. Directo, concentrado, cargado de razón, de razones. El reto es la acción rápida, técnicamente orientada, férreamente adherida a la información ágil, coordinada, eficaz y respetuosa con los procesos; estrechamente unida a la escucha, a la participación, a la toma de decisiones compartidas con el entorno usuario; tomándole en consideración, amparando su desarrollo como sistema, fortaleciendo sus mecanismos para el afrontamiento y el protagonismo responsable, digno y estable; facilitando la generación de un itinerario proactivo, orientado, optimista, abierto a la mejora permanente, de largo aliento, equilibrador y vivo.
El objetivo es la acción. La acción planificada, orientada. Y para todos. La delgada línea entre los que son y los que pueden ser no está justificada en este escenario. Porque no tiene sentido liquidar sin más la doctrina de la prevención en sus contenidos más técnicamente ligados a la idea de ayudar a que la alteración no se produzca. Porque no tiene sentido arrinconar, obviar u olvidar (mirar hacia otro lado, en suma) la acción prioritaria de evitar la perturbación cuando existe el riesgo o probabilidad de que ésta se instale; porque no podemos seguir soportando el coste, en no pocos casos, de abandonar a su suerte al riesgo, a la probabilidad, a la experiencia, técnicamente reconocida, de sucumbir al síntoma cuando éste ha podido evitarse. Porque no podemos despreciar lo que con tanta rotundidad informa, define y configura, sin ambages, la ciencia de la neurología evolutiva en las primeras edades.

Atención inmediata tras el diagnóstico de afectación o riesgo de la misma. Este es el norte, el objetivo a alcanzar. Los procedimientos tasados, por supuesto, Técnicamente avalados, claro. Doctrinalmente fundamentados, sí. Institucionalmente definidos y pautados, cómo no. Pero atención inmediata. Ágil. Orientada por, para y con todo el sistema usuario, el niño y su entorno. Actuación planificada, establecida en protocolos, en itinerarios claros y detallados.

La opción no puede ser otra. Los tres primeros años de vida, y, en especial, los primeros seis meses, son de tan vital importancia que cualquier otra orientación o interpretación más restrictiva se antoja banal, sesgada, escorada desde el origen. Tumbada en su desarrollo. Tardía en sus presupuestos, Defectuosa en su configuración. Tal vez sea preciso no perder de vista que estamos hablando de un problema de salud y de prevención de la salud, más allá de la consideración de si se tiene derecho o no a una prestación social que encaje con determinadas políticas de derivación a estos o aquellos servicios.

Negar la posibilidad de entender la atención temprana como un contexto de intervención propio de los catálogos de servicios de los sistemas sanitarios, en su más básica y prioritaria interpretación, no deja de ser una mirada lenta y desorientada de las necesidades y los derechos de la población a la que hacemos referencia.

Resultaría descorazonador que, precisamente, el resultado de políticas de gran calado y eficiencia en materia de prevención de la discapacidad, no encontraran su acomodo de desarrollo en las acciones que, sin reserva alguna, abundan en la mejora de la calidad de vida, presente y futura, de los beneficiarios de aquéllas. Resultaría, tal vez resulta, doloroso, que no fuéramos capaces como sistema de responder con destreza a los riesgos de una sociedad que, de una manera creciente, toma en consideración de forma tibia, por decir algo, y habilita, sin demasiada autorreflexión, situaciones suficientemente reconocidas en las que los partos 
prematuros, y sus consecuencias traducidas en riesgo de discapacidad o en discapacidad, avanzan cuanti y cualitativamente de forma imparable.

Insisto. La acción rápida, ágil, profesional, orientada, protocolizada, coordinada. Ahí está el reto. En todo el Estado. Con seguridad jurídica suficiente. Con criterio técnico e interinstitucional fundado y estable. Los procedimientos, administrativos o técnicos, para la concreción de principios básicos de la atención a prestar no deben condicionar el objetivo esencial de atender con premura, y, por ende, con la máxima eficiencia. A las edades a las que nos referimos (y, por tanto, en la coyuntura de desarrollo y maduración profusamente documentada científicamente) el tiempo vuela y las necesidades y derechos de los afectados precisan de cobertura y satisfacción a la mayor brevedad. Nos jugamos mucho.

Permítaseme incluir, asimismo, a este conjunto de ideas una que estimo imprescindible para dar coherencia a lo expuesto. Ya se ha esbozado con anterioridad. La acción definida y protocolizada desde el diagnóstico con cada niño. Un camino orientado y pactado con su entorno. Un sendero marcado, pautado con las intervenciones profesionales que se estimen precisas, apuntalado con la evaluación continua. Un proceso visible y entendible. Una idea ya apuntada por el CERMI en la Comisión Permanente no legislativa para las políticas integrales de la discapacidad ${ }^{10}$ de la Asamblea de Madrid en 2009. El itinerario personalizado para las personas con discapacidad.

"Desde que un niño o una niña nace empieza el gran peregrinaje de muchas familias que no saben qué hacer, familias a las que, de repente, se les viene el mundo encima porque no encuentran una coordinación secuencial sobre lo que hay que hacer o dónde ir para que su hijo sea bien atendido y se puedan reducir al máximo las consecuencias que se deriven de su situación inicial de discapacidad. Es necesario su reconocimiento dentro de un marco legal que lo regule como un derecho: es un procedimiento vital para la prevención o desarrollo de una discapacidad en su edad más temprana, una demanda de reivindicación histórica del colectivo que debe dar respuesta a las necesidades transitorias o permanentes que pueden presentar los menores con discapacidad en su desarrollo o que puedan tener el riesgo de padecerla. Debe ser éste un tratamiento de actuaciones integrales que den una rápida respuesta a través de una intervención inmediata, un diagnóstico y una intervención precoz" 11 .

El objetivo ha de trazar un recorrido razonable, definible con agilidad, revisable según las circunstancias y resultados procesuales, explicable, constructivo, ope-

\footnotetext{
${ }^{10}$ Comisión Permanente no legislativa para las políticas integrales de la discapacidad. Diarios de sesiones de la Asamblea de Madrid números 518, 537 y 563 .

11 (Diario de sesiones de la Asamblea de Madrid, 2009, $\mathrm{n}^{\circ} 563$ ).
}

rable con facilidad. La idea apunta a la necesidad de revisar el actual modelo de atención, excesivamente fragmentado, solapado en no pocas instancias, duplicado en otras tantas. Revisar la acción. Incardinar las acciones para la atención temprana sin actuaciones encapsuladas, alejadas de las disonancias y celotipias profesionales e institucionales. Un espacio, un lugar donde se gestione la acción tras el diagnóstico profesional. La acción en los diferentes ámbitos. Que analice necesidades y mecanismos para la acción preventiva, de habilitación y/o rehabilitación específicas. Que evalúe y asigne programas de acción individualizada. Con, y siempre con la participación y el protagonismo de las familias.

Es imprescindible ahondar de manera seria y responsable en la definición de los procesos que tienen lugar en los diferentes ámbitos (Casado, 2009) por los que han de transitar quienes se ven inmersos en el siempre complejo contexto de la atención temprana: (1) el escenario hospitalario: el acceso a la vida extrauterina, la promoción de la salud, la prevención de la discapacidad y el primer diagnóstico (GAT, 2011 $)^{12}$ y actuaciones en materia de atención a ésta y al riesgo; (2) los tiempos y posibilidades de atención, cuidado y educación de los hijos en el entorno familiar en situaciones de atención a la discapacidad o riesgo (Sanz, 2011); (3) los recursos y servicios especializados para la atención temprana: criterios de calidad homogéneos, modalidades flexibles de atención y colaboración y participación con y de las familias; (4) el entorno escolar y los retos significativos en el primer tramo del actual sistema educativo: toma en consideración de la atención temprana como escenario de intervención compensadora; y (5) el escenario social y la importancia de los modelos en la atención al discapacitado: atender la discapacidad desde su prevención.

\section{Algunas conclusiones}

1. La atención temprana, como prestación de servicios irrenunciable, requiere de un sustrato jurídico estable y uniforme que habilite las cuotas necesarias de garantía y sostenibilidad de recursos y procesos tasados de intervención especializada e integral, en sus componentes de interdisciplinar e interinstitucional.

2. El reto, la apuesta pasa por la consideración de la atención temprana como un derecho subjetivo, ligado a la prestación garantizada de un servicio de atención especializado e incorporado a un catálogo de servicios amplio, integral y coordinado.

12 Federación Estatal de Asociaciones de Profesionales de la Atención Temprana- GAT (2011). Mejora del proceso de comunicación del diagnóstico de trastorno en el desarrollo o discapacidad en la primera infancia. La primera noticia. 
3. Consecuentemente, parecería razonable abordar su sustrato normativo como derecho subjetivo, universal, basado en criterios de prescripción técnica.

4. Las actuaciones en contextos definidos como de riesgo, sin que medie especificación o diagnóstico de discapacidad, precisan de un soporte y estabilidad técnica y jurídica imprescindibles que garantice la atención inmediata en el marco de las actuaciones de prevención primaria y secundaria que son exigibles en materia de promoción de la salud.

5. La acción en el marco de la atención temprana debe plantearse en el contexto de un itinerario personal definido técnicamente, una vez establecido el diagnóstico, desde el que cohesionar y combinar las actuaciones en los diferentes ámbitos disciplinares e institucionales.

6. Los procedimientos de diagnóstico, prescripción y desarrollo del citado itinerario deben definirse de manera pronta, sin premura y con la inexcusable participación del entorno familiar del niño implicado.

7. La acción debe plantearse desde la óptica y consideración de los padres y el sistema familiar como agentes básicos e imprescindibles del desarrollo y crianza de sus hijos.

8. Negar la posibilidad de entender la atención temprana como un contexto de intervención propio de los catálogos de servicios de los sistemas sanitarios, en su más básica y prioritaria interpretación, representa una interpretación ambigua y desorientada de las necesidades y los derechos de la población a la que hacemos referencia.

9. La acción combinada entre instituciones debe ir más allá de la mera transferencia de información entre las mismas en el abordaje de cada uno de los casos atendidos. La opción más razonable y eficaz ha de encajarse en principios de acción definida y compartida, complementaria $\mathrm{y}$, en todo caso, sensible con la idea esencial de que quien ha de moverse en el proceso de atención integral y coordinada es el sistema y no el usuario.

10 La promoción de la salud y la prevención de la discapacidad en las primeras edades y, especialmente, en los dos primeros años de vida (con especial significación a la atención a las situaciones de riesgo), no pueden sino considerarse parte sustantiva y básica de la atención a la discapacidad.

\section{Extended Summary}

The development of early attention in recent years has been significant; its key concepts have been established, clarified and confirmed to the extent that there is now general agreement about them. Early attention has been defined as the set of interventions focused on the child from 0 up to 6 years old, but also focused on the family of that child as well as his or her environment, in an attempt to respond as early as possible to the temporary or permanent needs of children who present disorders in their development or that are at risk of suffering them.

These interventions, which must consider all aspects of the child, have to be planned by an interdisciplinary team of professionals. Experiences of joint work, professional discussions, white papers and academic publications have all contributed to defining the ideas, the arguments and the underlying philosophy of the process of definition and intervention. Contents of a nuclear nature have been invoked concerning areas such as the leadership and participation with and of the familysocial and family integration; free services, universality and equality of opportunities; interdisciplinary and high-level professional qualifications; and the coordination and decentralization and division of resources and services.

Although professionals in this field have generally been taking forward steps, I do not believe that they have been sufficient to fully understand each other and overcome the independence of the different areas that rule our procedures. In addition, we also find areas of work and intervention that have scarcely evolved in the last 20 years, the study of which has not yet been developed sufficiently.

Finally, in terms of the underlying content ideas that are involved, there are 3 important points which must be dealt with using common sense and conviction in order to put into place a satisfactory early attention intervention.

We must answer three basic questions: why we intervene, when and how.

- The recognition of users' rights (the movement toward the needs to the rights).

- Professional and rapid intervention for all those who need it (act correctly today not tomorrow, with everyone), and, of course

- The combined action of institutions and agents (real multi-institutional action, institutions giving up "power" in order to consistently giver priority to the role of the user of the system).

In summary, this article allows us to make the following considerations:

1. The advances made in terms of the recognition of rights in the area of early attention are significant. In recent years, the legal code has shown important changes in the rights and guarantees that the law provides. Nevertheless, we have only dealt with the surface of the issue and are now facing a great deal of essential change. Early attention requires a stable and uniform legal structure to achieve satis- 
factory levels of guarantees, as well as a specialized and global intervention process, in its interdisciplinary and multi-institutional components. In any case, the conceptualization of the legislative state enables and allows definition and regulation to take place in the framework of the normative development for which the autonomous communities (regions) are responsible.

2. Regulation of rights and benefits in health and education helps to improve the performance of intervention practices with children and their families. The social sphere needs to be optimized, adequately resourced, sustainable and uniform.

3. Actions without a diagnosis of disability require technical and legal support, provided in the context of interventions arising from defined protocols for primary and secondary prevention

4 The goal is the action, planned and oriented for everybody. It makes no sense to refrain from action when there is a risk of disability, when symptoms can be prevented. Such an attitude would mean disregarding neurological science at early ages.

5. It is important to define the procedures of the different fields:

a.The hospital area means the access to life and health, and the first information and action in the absence of health.

b.Timings and attention programs, care and raising of children in the family.

c.Attention specialized resources in early childhood

d.The school environment

e.The social area and the importance of models of care for the disabled

\section{Referencias}

Casado, D. (2009). Articulación de los apoyos para la infancia y de los servicios sanitarios, sociales y educativos. Recuperado de http://www.genysi.es /index.php? option $=$ com_remository\&Itemid $=32 \& f$ unc $=$ fileinfo $\& i d=313$
Casado, D. (2011). De la LISMI a la LAAD. Polibea, 100. Especial, VI a VIII.

Cruz, J. A. (1998). El concepto de derecho subjetivo en la teoría contemporánea del Derecho. (Tesis Doctoral). Universidad de Alicante. Recuperado de http://rua.ua.es/dspace/handle/10045/3401

Dunst, C. J. (2005). Framework for practicing evidence-based early childhood, intervention and family support. CASEinPoint, 1, 1-11. Recuperado de http://www.fippcase.org/case inpo int/caseinpoint_ voll_nol.pdf

Federación Estatal de Asociaciones de Profesionales de Atención Temprana. (2005). Libro Blanco de Atención Temprana. Documentos. 55/2005. Real Patronato sobre Discapacidad. Madrid.

Federación Estatal de Asociaciones de Profesionales de la Atención Temprana - GAT (2011). Mejora del proceso de comunicación del diagnóstico de trastorno en el desarrollo o discapacidad en la primera infancia. La primera noticia. Real Patronato sobre Discapacidad. Madrid.

Giné, C. (2009). Calidad de vida familiar: propuestas para repensar la atención temprana. Recuperado de http://www.genysi.es/index.php? option=com_remo sitory \& Itemid $=32 \&$ func $=$ fileinfo $\& i d=315$

Luengo J. A. (1995). Coordinación interinstitucional en Atención Temprana: ¿Un proceso imposible? Polibea, 36, 4-7.

Luengo, J. A., Torrego , J. C., y García, R. J., (1994). El proceso de evaluación en el ámbito de la atención temprana. Polibea, 30, 3-7.

Peterander, F. (2009). Influencia del entorno en la salud mental infantil e intervención temprana. Recuperado de http://www.genysi.es/index.php? option $=$ com_remository\&Itemid $=32 \&$ func $=$ filein fo\&id=334

Sanz, M. J. (2011). La discapacidad, factor de vulnerabilidad para una crianza saludable. Boletín del Real Patronato sobre Discapacidad, 71, 12-17.

Vilá, A. (2009). La Atención Temprana: del reconocimiento a la exigencia a través del derecho subjetivo. Situación actual en la legislación española. Recuperado de http://www.genysi.es/index. php? option= com_remository\&Itemid $=32 \&$ func $=$ fileinfo $\&$ id $=296$

Manuscrito recibido: 02/03/2012

Revisión recibida: 21/09/2012

Manuscrito aceptado: 04/10/2012 